\title{
Two pregnancies during a nine year period of amenorrhea - a rare presentation of Cushing's disease
}

\author{
H N Rajaratnam ${ }^{1}$, K Dharshini², Noel Somasundaram ${ }^{3}$, Himashi Kularathna ${ }^{4}$ \\ Sri Lanka Journal of Diabetes, Endocrinology and Metabolism 2013; 3: 25-28
}

\begin{abstract}
We describe a young lady with poorly controlled diabetes and prolonged period of amenorrhoea who had two children during that period. Further evaluation confirmed Cushing's disease and control of hypercortisolaemia with medical therapy improved her symptoms. She underwent transsphenoidal surgery and histology revealed a "pituitary adenoma". Though pregnancy is uncommon in Cushing's syndrome, this case reports the rare possibility of pregnancy in Cushing's syndrome even without treatment and unusually during a very long period of amenorrhoea.
\end{abstract}

Key words: Cushing's syndrome, amenorrhoea, pregnancy

\section{Introduction}

The diagnosis of Cushing's syndrome may present a great challenge to physicians. Menstrual irregularity, amenorrhoea and subfertility are common in these patients, due to suppression of secretion of gonadotropin-releasing hormone $(\mathrm{GnRH})$ by hypercortisolaemia. Because of this fertility is rare, although cases of Cushing's disease in pregnancy have been reported. Pregnancy during a period of amenorrhoea is rare and two pregnancies during a very prolonged period of amenorrhoea would be very rare. We report a young lady with Cushing's disease who presented with prolonged amenorrhea of nine years but had two children during that period.

\section{Case report}

A 31 year old lady with a history of amenorrhea of 9 years duration and poorly controlled diabetes was referred to us.

She attained menarche at the age of 12, had irregular periods with 30 to 40 days cycles till she married at the age of 21. She delivered her first child at the age of 22 and never had periods for the next nine years. During this amenorrhoic period she conceived twice and now has three children. Her youngest child is 3 years old. She had never used any contraceptive method and there were no pregnancy losses.

During these nine years she gained weight and noticed a change in her body habitus and facial appea- rance. These included pigmentation of the body, easy bruising, and increased hair growth on the face, arm and legs.

She was diagnosed as having gestational diabetes three years ago during her third pregnancy at 30 weeks of gestation. She was treated with insulin during pregnancy and the diabetes did not remit after delivery. Insulin and metformin were continued over the next three years. She had poor glycaemic control and recurrent breast abscesses, requiring many incision and drainage procedures. She gave a history of renal calculi and no history of steroid consumption.

On examination she was overweight with a BMI of 27(body weight: $65 \mathrm{~kg}$, height: $155 \mathrm{~cm}$ ). She had a round facies, facial plethora, a dorsocervical fat pad, thin skin (Figure 1), hirsutism with downy sideburns, increased hair on the upper lip and below the chin, limbs and chest scoring 14 on the Ferriman-Gallwey score (Figure 2). She also exhibited sparse scalp hair, without other features of virilization, such as temporal balding, deepening voice, male body habitus, or clitoral hypertrophy. There were ecchymotic patches in the limbs, pigmentation on the face and back of the neck, abdominal obesity, wide purple striae and proximal myopathy of upper and lower limbs. The blood pressure was 150/90 mmHg without cardiomegaly. There was no evidence of fungal infection of nails or skin or any oragnomegaly (Figure 3). Rest of the examination including sensory system, visual fields and fundi were normal. 


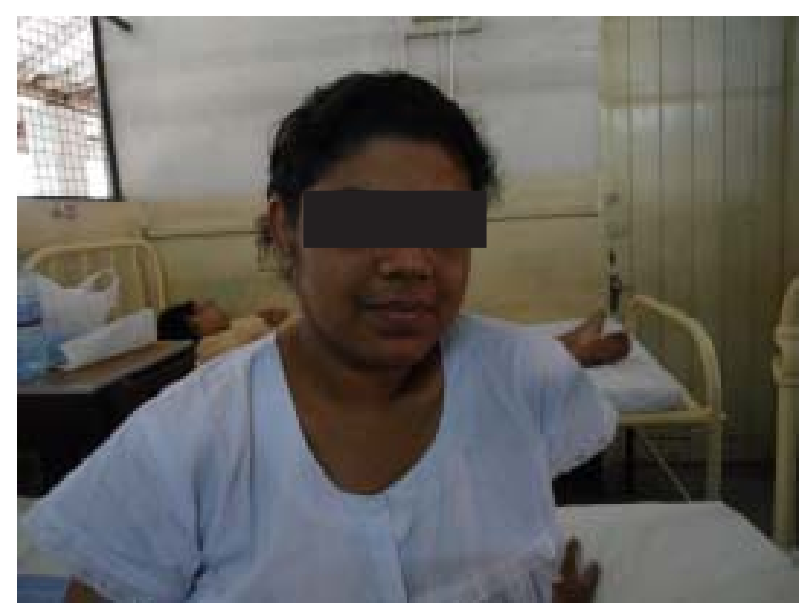

Figure 1. Patient showing round, plethoric "moon" face and pigmentation.

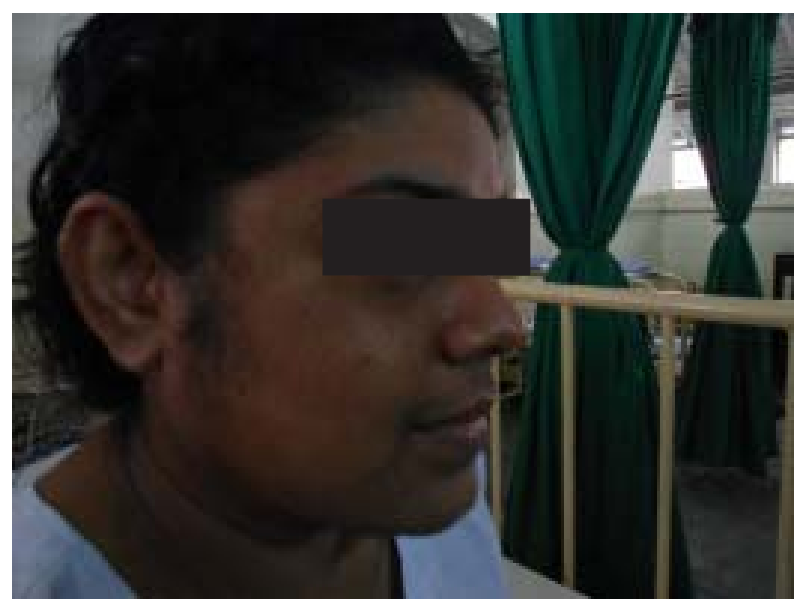

Figure 2. Hirsutism, downy sideburns and increased hair on the upper lip and under the chin.

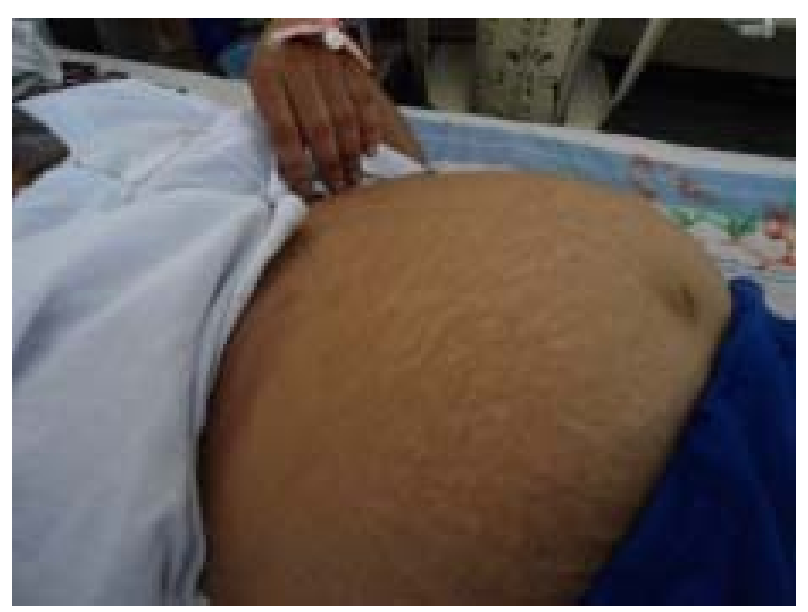

Figure 3. Abdominal obesity and lower abdominal purple striae.
The following tests were done and the results are given below.

1. Overnight dexamethasone suppression test (ODST): serum cortisol of $518 \mathrm{nmol} / \mathrm{l}$ (normal < 50nmol/l).

2. Low dose dexamethasone suppression test (LDDST): basal cortisol of $1160 \mathrm{nmol} / \mathrm{l}$ and after 48 hours, 1040nmol/l (not suppressed).

3. Adreno cortico trophic hormone (ACTH) of 92.9pg/ $\mathrm{ml}$ (normal76 pg/ml).

4. High-dose dexamethasone suppression test (HDDST): basal cortisol of 1060nmol/l and after 48 hours, $696 \mathrm{nmol} / \mathrm{l}$ (partially suppressed).

These confirmed hypercortisolism and partial suppression in HDDST. These results indicate ACTH dependent Cushing's syndrome or rarely Cushing's disease which presents with non suppression with high dose dexamethasone in $10 \%$ of cases (1). Magnetic resonance imaging of pituitary did not reveal any pituitary or hypothalamic lesions and computed tomography (CT) of chest, abdomen and pelvis were normal. As no information from these imaging procedures allowed localization, bilateral inferior petrosal sinus sampling (IPSS) was undertaken (Table 1). This revealed a central to peripheral gradient of 12.7 , suggesting the pituitary to be the source of the excess ACTH indicating a secreting pituitary tumour. A left to right gradient of 14.2 in ACTH lateralized the source to the left side of the pituitary (2). Other investigations including serum calcium and inorganic phosphate, 24 hour urinary calcium, 2D Echocardiogram and other pituitary hormonal assessments (Table 2) were normal. The DXA scan revealed a Z score in the osteoporosis range. Based on these investigations, a diagnosis of Cushing disease due to a pituitary micro adenoma was made. Diabetes mellitus, hypertension, osteoporosis, recurrent abscesses and renal calculi were attributed to the Cushing's disease (3).

After thorough discussion with the patient, trans sphenoidal surgery (TSS) was offered and accepted by the patient (4). While awaiting surgery, she was treated with ketoconazole $200 \mathrm{mg} 8$ hourly initially and cabergoline $0.5 \mathrm{mg}$ titrated upwards to $1.5 \mathrm{mg}$ weekly was added because of inadequate response $(5,6,7,8)$. Her diabetes was managed with insulin and metformin. During this period of medical management she developed periods for the first time in nine years. Thereafter she underwent TSS and histology revealed a "pituitary adenoma”. 
Table 1.

\begin{tabular}{lll}
\hline & ACTH(pg/ml) & Prolactin(mU/l) \\
\hline Right petrosal sinus & 109 & 87 \\
Left petrosal sinus & $>1550$ & 412 \\
Right internal jugular vein & 149 & 169 \\
Left internal jugular vein & 667 & 224 \\
Right brachio cephalic vein & 289 & \\
Left brachio cephalic vein & 259 & 163 \\
Superior vena cava & 161 & 136 \\
Femoral vein & 122 & \\
\hline
\end{tabular}

(Normal ranges: ACTH 10-50 $\mathrm{pg} / \mathrm{ml}$; Prolactin $0-460 \mathrm{mU} / \mathrm{l})$

Table 2.

\begin{tabular}{lll}
\hline Test & Result & Normal range \\
\hline FSH(mu/l) & 6.8 & $1-10$ \\
LH(mu/l) & 4.1 & $1-10$ \\
Prolactin (ng/dl) & 12 & $<25$ \\
Plasma total testosterone (ng/dl) & 0.43 & $<5$ \\
TSH(miu/) & 0.9 & $0.4-4$ \\
FT4 (ng/dl) & 1.4 & $0.8-1.8$ \\
& & \\
Serum calcium (mg/dl) & 9.00 & $2.8-10.3$ \\
Serum inorganic phosphate (mg/dl) & 3.20 & \\
24h urine volume (l) & 2.1 & $<250 \mathrm{mg} / \mathrm{day}$ \\
24h urinary calcium (mg) & 287.7 & \\
& & \\
DEXA scan (Z score) & & \\
Total spine & -3.4 & \\
Right hip & -0.2 & \\
Left hip & -0.7 & \\
2D Echo & Normal study & \\
\hline
\end{tabular}

\section{Discussion}

Cushing's syndrome is characterized by an array of clinical features that suggest the presence of hypercortisolism. Some patients may present with only isolated symptoms and in some the most common findings (truncal obesity and hypertension) may be absent (9). Cushing's syndrome may be misdiagnosed for a long time and patients might be treated in various clinics before the correct diagnosis is recognized. Depression is an atypical clinical presentation of Cushing's syndrome.

This patient was also treated for diabetes and renal stones for a significant period before the correct diagnosis was made. The diabetes is due to the tendency of glucocorticoids to increase insulin resistance in both liver and skeletal muscle. Renal calculi are attributed to the 
reduction of calcium absorption in the renal tubules leading to hypercalciuria. Osteoporosis is attributed to decreased bone formation. The period of amenorrhea for nine years could be explained by suppression of secretion of gonadotropin-releasing hormone $(\mathrm{GnRH})$ and inhibition of pulsatile secretion of luteinizing hormone ( $\mathrm{LH}$ ) and follicle-stimulating hormone (FSH) due to the hypercortisolaemia. Since ACTH secretion by these tumours are often intermittent, there may be periods during which the female hormones are insufficiently suppressed to prevent ovulation leading to pregnancy. In this patient, probably the levels of cortisol did not prevent ovulation but was sufficient to cause amenorrhea. This hypothesis is corroborated by the fact that GnRH neurons are known to express glucocorticoid receptors (10), indicating a possible mechanism by which hypercortisolemia can block GnRH/ gonadotropin release and be responsible for the menstrual disorders associated with Cushing's syndrome. Further corroboration was demonstrated as the patient developed periods for the first time when the cortisol levels were decreased with a combination of ketoconazole and cabergoline.

\section{Conclusion}

The association between Cushing's syndrome and pregnancy is uncommon due to associated sub-fertility. This case reports the rare possibility of pregnancy in Cushing's syndrome even without treatment. We have put forward a hypothesis for the mechanism by which pregnancy is possible during a prolonged period of amenorrhea in a patient with Cushing's disease.

\section{References}

1. Nieman LK, Biller BM, Findling JW, et al. The diagnosis of Cushing's syndrome: an Endocrine Society Clinical Practice Guideline. J Clin Endocrinol Metab 2008; 93: 1526.
2. Selective use of Bilateral Inferior Petrosal Sinus Sampling in Patients with Adrenocorticotropin-Dependent Cushing's Syndrome Prior to Transsphenoidal Surgery Sigrid Jehle, Jane E. Walsh, Pamela U. Freda, and Kalmon D. Post. J Clin Endocrinol Metab 2008; 93(12): 4624-4632.

3. Arnaldi G, Angeli A, Atkinson AB, et al. Diagnosis and complications of Cushing's syndrome: a consensus statement. J Clin Endocrinol Metab 2003; 88: 5593-602.

4. James W. Findling and Hershel Raff, Clinical review: Cushing's Syndrome: Important Issues in Diagnosis and Management The Journal of Clinical Endocrinology and Metabolism 2006; 91(10): 3746-53.

5. Tritos Beverly MK, Biller BS, Nicholas A. Management of Cushing Disease: Managing Persistence or Recurrence. Medscape, CME Released: 02.08.2011.

6. de Bruin C, Feelders R A, Lamberts SW, Hofland LJ. Somatostatin and dopamine receptors as targets for medical treatment of Cushing's Syndrome. Rev. Endocr. Metab. Disord. 2009; 10: 91-102.

7. Pivonello R. et al. The medical treatment of Cushing's disease: effectiveness of chronic treatment with the dopamine agonist cabergoline in patients unsuccessfully treated by surgery. J. Clin. Endocrinol. Metab. 2009; 94: 223-30.

8. Nieman L K. Medical therapy of Cushing's disease. Pituitary 2002; 5: 77-82.

9. Marco Boscaro MD, Luisa Barzon MD, Nicoletta Sonino, MD. The Diagnosis of Cushing's Syndrome - Atypical Presentations and Laboratory Shortcomings. Arch Intern Med. 2000; 160(20): 3045-53.

10. Ahima RS, Harlan RE. Glucocorticoid receptors in LHRH neurons. Neuroendocrinology 1992; 56: 845-50. 